\title{
Focal shift of radially polarized bessel-modulated gaussian beam by phase shifting
}

\author{
Xiu-Min $\mathrm{Gao}^{1,2}$, Ming-Yu Gao', Song Hu${ }^{1}$, Han-Ming Guo ${ }^{2}$, Jian Wang ${ }^{1}$, Song-Lin Zhuang ${ }^{2}$ \\ ${ }^{1}$ Electronics \& Information College, Hangzhou Dianzi University, Hangzhou, China; xiumin gao@yahoo.com.cn \\ ${ }^{2}$ Optics \& Electronics College, University of shanghai for Science and Technology, Shanghai, China
}

Received 28 September 2009; revised 23 October 2009; accepted 26 October 2009.

\begin{abstract}
Focal shift of radially polarized Bessel-modulated Gaussian (QBG) beam by phase shifting is investigated theoretically by vector diffraction theory. The phase shifting distribution is the function of the radial coordinate. Calculation results show that intensity distribution in focal region can be altered considerably by the topological charge of QBG beam and the phase parameter that indicates the vary degree of the phase shifting along radial coordinate. Topological charge induces the focal shift in transverse direction, while phase parameter leads to the focal shift along optical axis of the focusing system. More interesting, the focal shift may be incontinuous in certain case.
\end{abstract}

Keywords: Focal Shift; Bessel-Modulated Gaussian Beam; Vector Diffraction Theory

\section{INTRODUCTION}

Since Caron and Potvliege introduced a novel class of beam expressed in cylindrical coordinate system recently, namely, the Bessel-modulated Gaussian beams with quadratic radial dependence (QBG beam) [1], QBG beam has attracted much attention [2-7]. It was shown that such class of beams has familiar collinear geometry of the Gaussian beam and also an interesting non-Gaussian features for certain values of its parameters [1-3]. Belafhal and Dalil-Essakali studied the propagation properties of QBG beams through a unapertured optical paraxial ABCD system [4]. X. Wang, and B. Lü researched on the beam propagation factor $\left(\mathrm{M}^{2}\right.$-factor), far-field distribution, and the kurtosis parameter of such type of beams $[3,5,6]$. And the Bessel-modulated Gaussian light beams passing through a paraxial ABCD optical system with an annular aperture has also been studied [7]. On the other hand, in the investigation of the focusing properties of optical beams, tracing the movement of the point of absolute maximum intensity along optical axis has attracted many researchers for several decades [8-12]. It was found that the point of absolute maximum intensity does not coincide with the geometrical focus but shifts along optical axis. This phenomenon is referred to as focal shift. More interesting, the focal shift may be incontinuous in certain optical focusing systems.

Almost all QBG beams in above previous papers are in scalar a form, which means the polarization property of optical field is not considered. In fact, the polarization is very important characteristics to alter propagating and focusing properties of beams. For example, laser beam with cylindrical symmetrical polarization have attracted many researchers recently because the electric field in focal region of such cylindrical vector beam has some unique properties [13-16]. The present paper is aimed at studying focal shift of radially polarized QBG beam by vector diffraction theory. The principle of the focusing radially polarized QBG beam with phase shifting is given in Section 2. Section 3 shows the simulation results and discussions. The conclusions are summarized in Section 4.

\section{PRINCIPLE OF THE FOCUSING RADIALLY POLARIZED QBG BEAM WITH PHASE SHIFTING}

In the focusing system we investigated, focusing beam is radially polarized QBG beam whose value of transverse optical field is same as that of the scalar QBG [1-3], and its polarization distribution turns on radially symmetric $[13,14]$. Therefore, in the cylindrical coordinate system $(r, \varphi, 0)$ the field distribution $\vec{E}(r, \varphi, 0)$ of the radially polarized QBG beam at the plane is written as,

$$
\vec{E}_{0}(r, \phi, 0)=E_{0}(r, \phi, 0) \cdot \vec{n}_{r}
$$

where $\vec{n}_{r}$ is the radial unit vector of polarized direction. Term $\vec{E}(r, \varphi, 0)$ is optical field value distribution and can be written in the from [1-5], 


$$
E_{0}(r, \phi, 0)=B \cdot J_{|m| / 2}\left(\frac{\mu r^{2}}{\omega_{0}^{2}}\right) \exp \left(-\frac{r^{2}}{\omega_{0}^{2}}\right) \exp (i m \phi)
$$

where $J_{|m| / 2}$ denotes the Bessel function of order $|\mathrm{m}| / 2$, $m$ is the topological charge of QBG beam, $\omega_{0}$ is the waist width of the Gaussian beam, and $\mu$ is a beam parameter which is complex-valued in general. $B$ is a constant. According to vector diffraction, the electric field in focal region of radially polarized QBG beam is [17],

$$
\vec{E}(\rho, \varphi, z)=E_{\rho} \vec{e}_{\rho}+E_{\varphi} \vec{e}_{\varphi}+E_{z} \vec{e}_{z}
$$

where $\vec{e}_{\rho}, \vec{e}_{\varphi}$, and $\vec{e}_{z}$ are the unit vectors in the radial, azimuthal, and propagating directions, respectively. To indicate the position in image space, cylindrical coordinates $(\rho, \varphi, z)$ with origin $\rho=z=0$ located at the paraxial focus are employed. $E_{\rho}, E_{\mathrm{z}}$, and $E_{\phi}$ are amplitudes of the three orthogonal components and can be expressed as.

$$
\begin{gathered}
E_{\rho}(\rho, \varphi, z)=\frac{-i A}{\pi} \int_{0}^{\alpha} \int_{0}^{2 \pi} \sqrt{\cos \theta} \cdot E_{0} \cdot \sin \theta \cos \theta \cos (\phi-\varphi) \\
\cdot \exp \{i k[z \cos \theta+\rho \sin \theta \cos (\phi-\varphi)]\} d \phi d \theta \\
E_{\varphi}(\rho, \varphi, z)=\frac{-i A}{\pi} \int_{0}^{\alpha} \int_{0}^{2 \pi} \sqrt{\cos \theta} \cdot E_{0} \\
\cdot \sin \theta \cos \theta \sin (\phi-\varphi) \\
\cdot \exp \{i k[z \cos \theta+\rho \sin \theta \cos (\phi-\varphi)]\} d \phi d \theta \\
E_{Z}(\rho, \varphi, z)=\frac{i A}{\pi} \int_{0}^{\alpha} \int_{0}^{2 \pi} \sqrt{\cos \theta} \cdot E_{0} \cdot \sin ^{2} \theta
\end{gathered}
$$

where $\theta$ and $\varphi$ denote the tangential angle with respect to the $z$ axis and the azimuthal angle with respect to the $x$ axis, respectively. $\mathrm{k}$ is wave number. $\alpha=\arcsin (N A)$ is convergence angle corresponding to the radius of incident optical aperture. In order to make focusing properties clear and simplify calculation process, after simple derivation, Eq. 2 can be rewritten as,

$$
\begin{gathered}
E_{0}(\theta, \phi, 0)=B \cdot J_{|m| / 2}\left[\frac{\sin ^{2}(\theta)}{w^{2} \cdot N A^{2}}\right] \\
\cdot \exp \left[-\frac{\sin ^{2}(\theta)}{w^{2} \cdot N A^{2}}\right] \cdot \exp (i m \phi)
\end{gathered}
$$

where $w=\omega_{0} / r_{0}$ is called relative waist width. The phase shifting of the radially polarized QBG beam is the function of radial coordinate and is in the from as,

$$
\psi=\pi \cdot \cos \left(C \cdot \frac{\tan \theta}{\tan \alpha} \cdot \pi\right)
$$

where $C$ is phase parameter that indicates the vary de- gree of the phase shifting along radial coordinate. Substitute the Eq.7 and Eq.8 into Eqs.4-6, we can obtain,

$$
\begin{aligned}
& E_{\rho}(\rho, \varphi, z)=\frac{-i A B}{\pi} \int_{0}^{\alpha} \int_{0}^{2 \pi} \sqrt{\cos \theta} \cdot \sin \theta \cos \theta \\
& \cdot \cos (\phi-\varphi) \cdot J_{|m| / 2}\left[\frac{\sin ^{2}(\theta)}{w^{2} \cdot N A^{2}}\right] \cdot \exp \left[-\frac{\sin ^{2}(\theta)}{w^{2} \cdot N A^{2}}\right] \\
& \cdot \exp (i \psi) \cdot \exp (i m \phi) \cdot \exp \left[i \cdot \pi \cdot \cos \left(C \cdot \frac{\tan \theta}{\tan \alpha} \cdot \pi\right)\right] \\
& \cdot \exp \{i k[z \cos \theta+\rho \sin \theta \cos (\phi-\varphi)]\} d \phi d \theta \\
& E_{\varphi}(\rho, \varphi, z)=\frac{-i A B}{\pi} \int_{0}^{\alpha} \int_{0}^{2 \pi} \sqrt{\cos \theta} \cdot \sin \theta \cos \theta \\
& \cdot \sin (\phi-\varphi) \cdot J_{|m| / 2}\left[\frac{\sin ^{2}(\theta)}{w^{2} \cdot N A^{2}}\right] \cdot \exp \left[-\frac{\sin ^{2}(\theta)}{w^{2} \cdot N A^{2}}\right] \\
& \cdot \exp (i \psi) \cdot \exp (i m \phi) \cdot \exp \left[i \cdot \pi \cdot \cos \left(C \cdot \frac{\tan \theta}{\tan \alpha} \cdot \pi\right)\right] \\
& \cdot \exp \{i k[z \cos \theta+\rho \sin \theta \cos (\phi-\varphi)]\} d \phi d \theta \\
& E_{Z}(\rho, \varphi, z)=\frac{i A B}{\pi} \int_{0}^{\alpha} \int_{0}^{2 \pi} \sqrt{\cos \theta} \cdot \sin ^{2} \theta \cdot \exp (i \psi) \\
& \cdot J_{|m| / 2}\left[\frac{\sin ^{2}(\theta)}{w^{2} \cdot N A^{2}}\right] \cdot \exp \left[-\frac{\sin ^{2}(\theta)}{w^{2} \cdot N A^{2}}\right] \cdot \exp (i m \phi) \\
& \cdot \exp \left[i \cdot \pi \cdot \cos \left(C \cdot \frac{\tan \theta}{\tan \alpha} \cdot \pi\right)\right] \\
& \cdot \exp \{i k[z \cos \theta+\rho \sin \theta \cos (\phi-\varphi)]\} d \phi d \theta
\end{aligned}
$$

The optical intensity in focal region is proportional to the modulus square of Eq.3. Basing on the above equations, focusing properties of radially polarized QBG beam with phase shifting can be investigated theoretically.

\section{RESULTS AND DISCUSSIONS}

Without of loss of validity and generality, it was supposed that $N A=0.95, \mu=5$ and $w=1$. Firstly, the intensity distributions in focal region of the radially polarized QBG beam with phase shifting are calculated under condition of $m=0$ and different $C$, and are illustrated in Figure 1. It should be noted that the distance unit in all figures in this paper is $k^{-1}$, where $k$ is the wave number of incident beam. In addition, the coordinates are the radial distance and axial distance, and symmetric characteristics should be paid attention to when see figures. It can be seen that there occurs one dark hollow focus in focal region for $C=0.0$. Dark hollow focus refers to those focuses whose optical intensity is weaker than that around it and is stable optical trap for those particles 
whose refractive index is smaller than that of surrounding media, and this condition is very common, especially in life science optical trapping systems, so construction of dark focal spot is very important and attracts many researchers $[18,19]$. Therefore, radially polarized QBG beam can be used to construct dark hollow focus. On increasing $C$, this dark hollow focus shifts along optical axis away from the optical aperture of the focusing system, as shown in Figure 1b. And the point of absolute maximum intensity also shifts along optical axis. The number of the absolute maximum intensity changes from

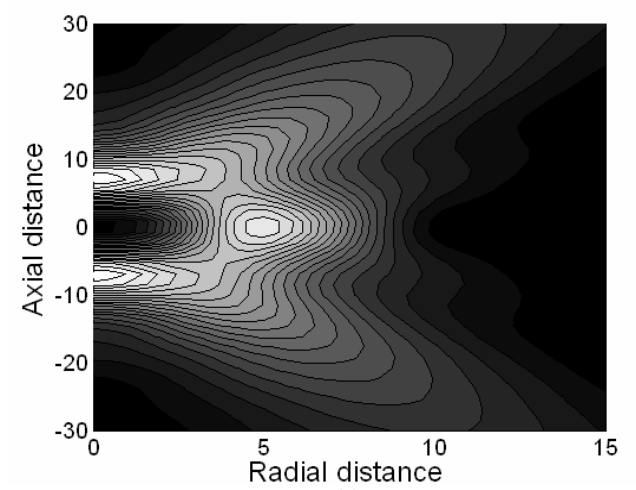

(a)

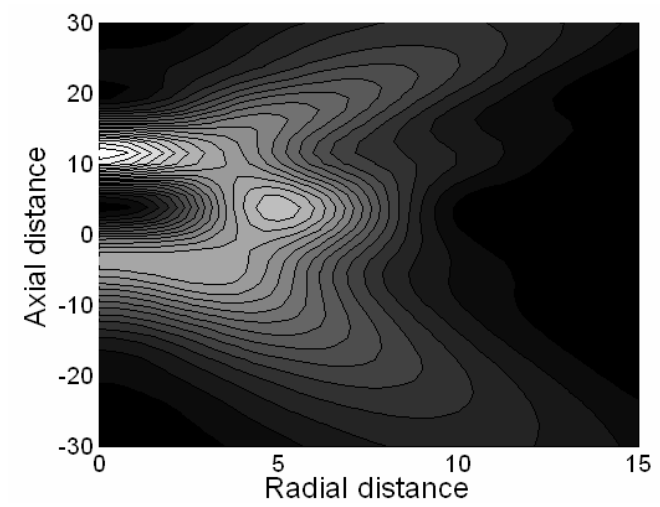

(c)

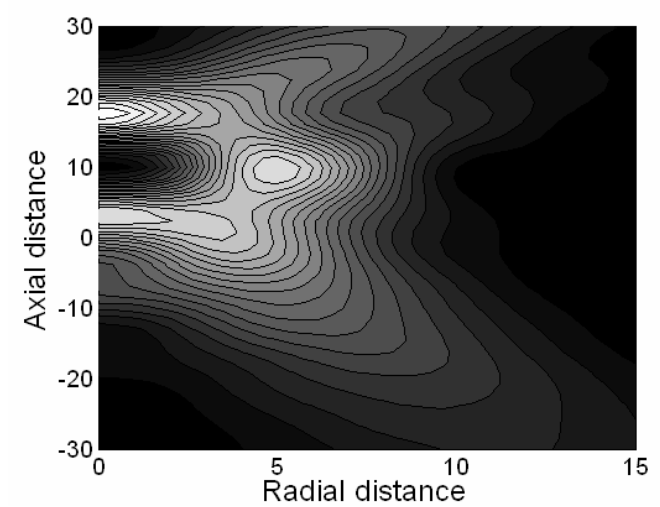

(e) two to one, namely, the on-axis intensity peak near to the optical aperture weakens on increasing $C$, as illustrated in Figure 1c. Increase $C$ continuously, the shape of dark hollow focus goes on shifting along optical axis. How ever, the position of absolute maximum intensity becomes incontinuous, namely, jumps to one position near the optical aperture, and then also shifts far away from optical aperture.

In order to understand the focal shift deeply, the dependence of focal shift on $C$ is calculated and shown in

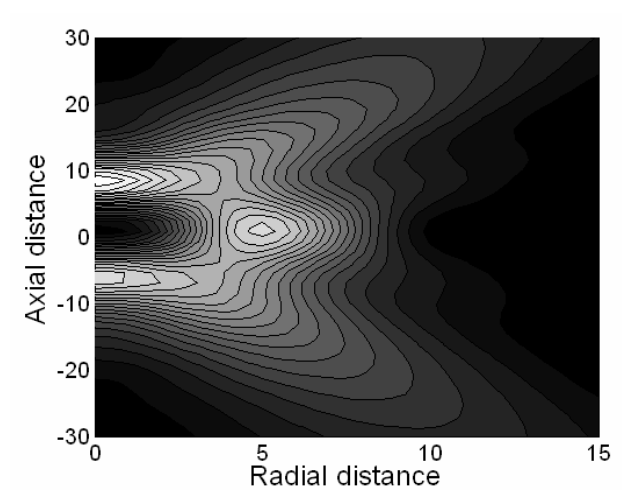

(b)

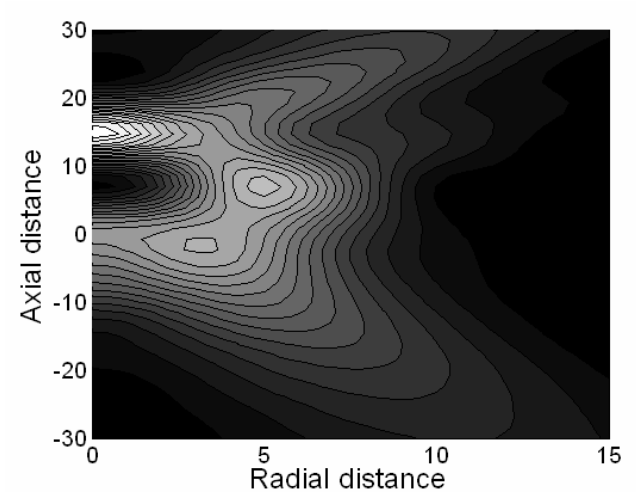

(d)

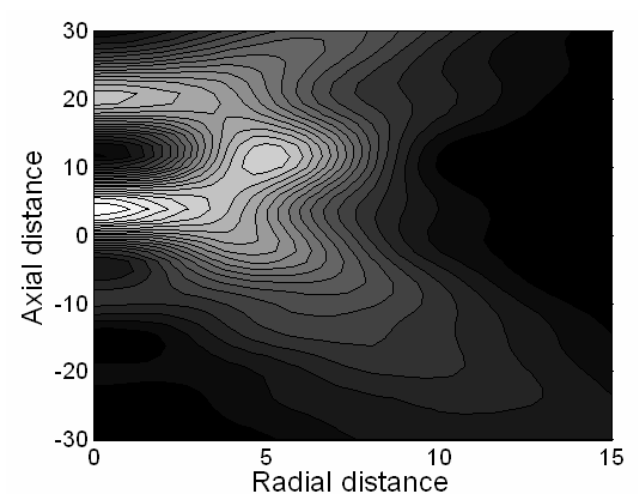

(f)

Figure 1. Intensity distributions in focal region for $m=0$ and (a) $C=0.0$, (b) $C=0.3$, (c) $C=0.6$, (d) $C=0.9$, (e) $C=1.2$, and (f) $C=1.5$, respectively 
Figure 2. It can be seen from this figure that when $C=0.0$ there are two absolute maximum intensity peaks on axis, and on increasing $C$, one absolute maximum intensity peaks weakens so that there is only one absolute maximum intensity peak, and in the focal evolution process, the distance of focal shift increases on increasing $C$.

When $C$ changes from 1.2 to 1.3 , the position of absolute maximum intensity peak jumps from on position to another position, then also shifts increases on increasing $C$. Focal shift is incontinuous.

Figure 3 illustrates the optical intensity distributions in focal region under condition of $m=2$ and different $C$.

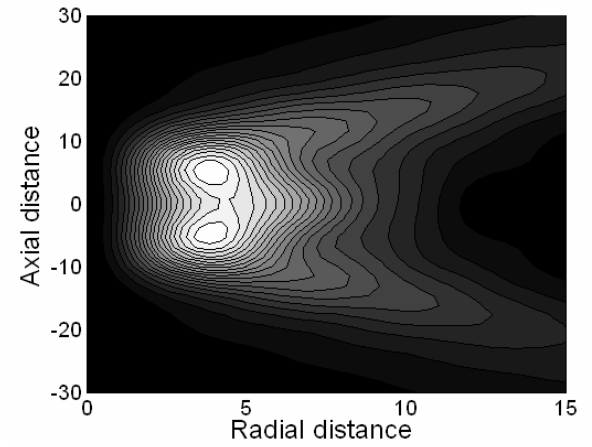

(a)

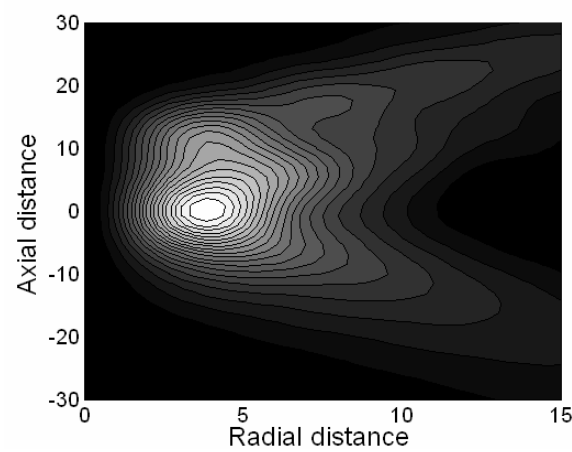

(c)

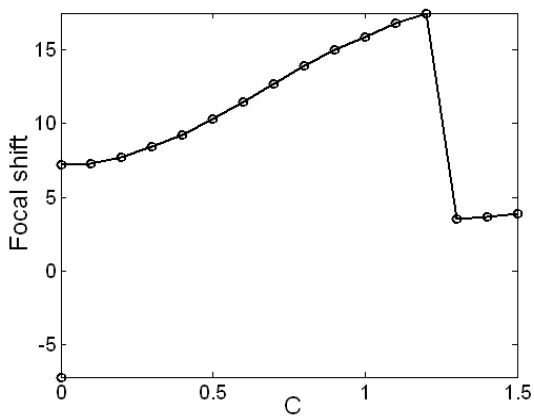

Figure 2. Dependence of focal shift on $C$ for $m=0$.

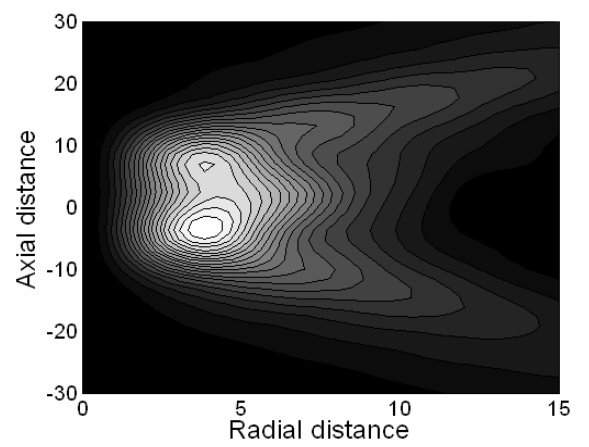

(b)

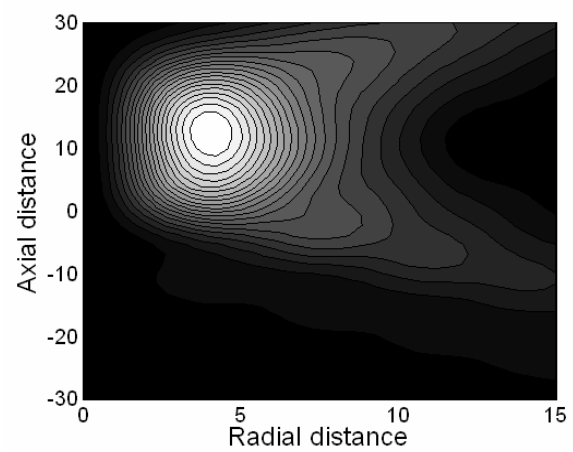

(d)

Figure 3. Intensity distributions in focal region for $m=2$ and (a) $C=0.0$; (b) $C=0.3$; (c) $C=0.6$, and; (d) $C=1.5$, respectively.

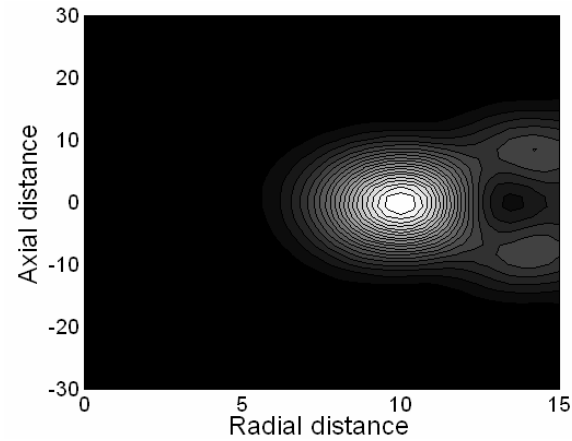

(a)

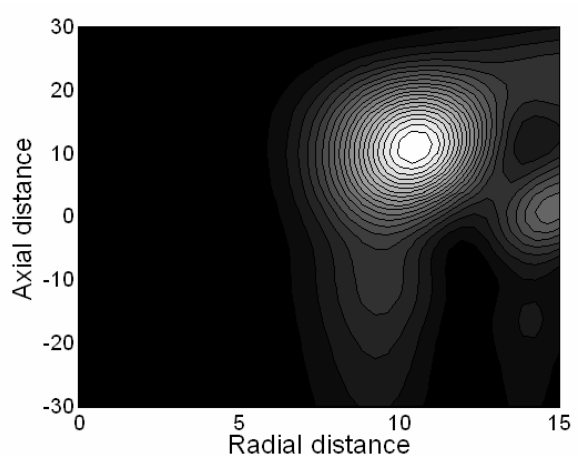

(b)

Figure 4. Intensity distributions in focal region for $m=7$ and (a) $C=0.0$; (b) $C=1.5$, respectively. 
For $C=0.0$, there is two overlapping intensity rings in focal region, as shown in Figure 3a. On increasing $C$. one of these two intensity ring weakens, so that one focal ring comes into being and shifts in axial direction. From all above focal pattern evolution, we can see that Topological charge induces the focal shift in transverse direction, while phase parameter leads to the focal shift along optical axis of the focusing system. In order to show this point, optical intensity distributions in focal region under condition of $m=7$ are also calculated and illustrated in Figure 4. Ring intensity distribution can be used to construct a ring optical trap that is stable for those particles in focal region whose refraction index is bigger than that of their surrounding medium.

\section{CONCLUSIONS}

Focal shift of radially polarized QBG beam by phase shifting is investigated theoretically by vector diffraction theory in this paper. The phase shifting distribution is the function of the radial coordinate. Simulations results show that intensity distribution in focal region can be altered considerably by the topological charge of QBG beam and the phase parameter that indicates the vary degree of the phase shifting along radial coordinate. Dark hollow focus can be obtained in focal region of radially polarized QBG beam, which is very desirable in optical tweezers technique. Particularly, topological charge induces the focal pattern evolution in transverse direction, while phase parameter leads to the focal shift along optical axis more significantly.

\section{ACKNOWLEDGMENT}

This work was supported by National Basic Research Program of China (2005CB724304), National Natural Science Foundation of China (60708002, 60777045, 60871088, 60778022), China Postdoctoral Science Foundation (20080430086), and Shanghai Postdoctoral Science Foundation of China (08R214141).

\section{REFERENCES}

[1] C. F. R. Caron and R. M. Potvliege, (1999) Bessel-modulated Gausian beams with quadratic radial dependence. Opt. Commun, 164, 83-93.

[2] Z. Hricha and A. Belafhal, (2005) Focal shift in the axisymmetric Bessel-modulated Gaussian beam. Opt. Commun, 255, 235-240.
[3] X. Wang and B. Lü, (2002) The beam propagation factor and far-field distribution of Bessel-modulated Gaussian beams. Opt. Quant. Electron, 34, 1071-1077.

[4] A. Belafhal and L. Dalil-Essakali, (2000) Collins formula and propagation of Bessel-modulated Gaussian light beams through an ABCD optical system. Opt. Commun, 177, 181-188.

[5] X. Wang and B. Lü, (2003) The beamwidth of Besselmodulated Gaussian beams. J. Mod. Opt, 50, 2107-2115.

[6] B. Lü and X. Wang, (2002) Kurtosis parameter of Bessel-modulated Gaussian beams propagating through ABCD optical systems. Opt. Commun, 204, 91-97.

[7] Z. Mei, D. Zhao, X. Wei, F. Jing, and Q. Zhu, (2005) Propagation of Bessel-modulated Gaussian beams through a paraxial ABCD optical system with an annular aperture. Optik, 116, 521-526.

[8] Y. Li and E. Wolf, (1981) Focal shifts in diffracted converging spherical waves. Opt. Commun, 39, 211-215.

[9] M. M. Corral, M. T. Caballero, L. M. Escriva, and P. Andres, (2001) Focal-shift formula in apodized nontelecentric focusing systems. Opt. Lett, 26, 1501- 1503.

[10] C. J. R. Sheppard and P. Tötök, (2003) Focal shift and axial coordinate for high-aperture systems of finite Fresnel number. J. Opt. Soc. Am. A, 20, 2156-2162.

[11] X. Gao, F. Zhou, W. Xu, and F. Gan, (2005) Gradient force pattern, focal shift, and focal switch in an apodized optical system. Optik, 116, 99-106.

[12] X. Gao and J. Li, (2007) Focal shift of apodized truncated hperbolic-Cosine-Gaussian beam. Opt. Commun, 273, 21-27.

[13] K. S. Youngworth and T. G. Brown, (2000) Focusing of high numerical aperture cylindrical-vector beams. Optics Express, 7, 77-87.

[14] Q. Zhan, (2009) Cylindrical vector beams: from mathematical concepts to applications. Advances in Optics and Photonics, 1, 1-57.

[15] X. Gao, S. Hu, H. Gu, and J. Wang, (2009) Focal shift of three-portion concentric piecewise cylindrical vector beam. Optik, 120, 519-523.

[16] F. Lu, W. Zheng, and Z. Huang, (2009) Coherent antistokes Raman scattering micriscopy using tighly focused radially polarized light. Opt. Lett, 34, 1870-1871.

[17] S. Sato and Y. Kozawa, (2009) Hollow vortex beams. J. Opt. Soc. Am. A, 26, 142-146.

[18] J. Arlt and M. J. Padgett, (2000) Generation of a beam with a dark focus surrounded by regions of higher intensity: The optical bottle beam. Opt. Lett, 25, 191193.

[19] D. Ganic, X. Gan, M. Gu, M. Hain, S. Somalingam, S. Stankovic, and T. Tschudi, (2002) Generation of doughnut laser beams by use of a liquid-crystal cell with a conversion efficiency near 100\%. Opt. Lett, 27, 13511353. 